\title{
Application of a $n$-Phase Model to the Diffusion Coefficient of Chloride in Mortar
}

\author{
S. CARÉ ${ }^{1, *}$ and E. HERVÉ 2 \\ ${ }^{1}$ Laboratoire Central des Ponts et Chaussées, Paris, France \\ ${ }^{2}$ Laboratoire de Mécanique des Solides, Ecole Polytechnique, Université de Versailles, Saint \\ Quentin en Yvelines, France
}

\begin{abstract}
The determination of the chloride diffusion coefficient of a concrete is needed to help the prediction of the service life of concrete structure. In this paper, we propose first a critical review of models for chloride diffusion coefficients already used in literature at different scales and then we develop an analytical model, which takes into account the characteristics of the different phases of concrete. These materials are treated as a three-phase composite, consisting of a cement continuous phase, of an aggregates dispersed phase and of an interface transition zone. Chloride diffusion coefficient using an $n$-layered inclusion-based micromechanical modeling is predicted. The details of calculations are summarized hereafter and experimental measurements obtained on mortars are compared with predicted results.
\end{abstract}

Key words: mortar, chlorides, diffusion, effective medium theory, homogenization technique, modeling.

\section{Nomenclature}

c volume fraction of ions $\left(\mathrm{kg} / \mathrm{m}^{3}\right)$.

$C_{\mathrm{A}} \quad$ volume fraction of aggregates (-).

$C_{\mathrm{I}} \quad$ volume fraction of ITZ (-).

D diffusion coefficient tensor in composite media $\left(\mathrm{m}^{2} / \mathrm{s}\right)$.

$D_{\mathrm{A}} \quad$ diffusion coefficient of ions in aggregates $\left(\mathrm{m}^{2} / \mathrm{s}\right)$.

$D_{\mathrm{B}} \quad$ diffusion coefficient of ions in bulk cement paste $\left(\mathrm{m}^{2} / \mathrm{s}\right)$.

$D_{i} \quad$ diffusion coefficient of ions in phase $i\left(\mathrm{~m}^{2} / \mathrm{s}\right)$.

$D_{\mathrm{P}} \quad$ diffusion coefficient of ions in cement paste $\left(\mathrm{m}^{2} / \mathrm{s}\right)$.

$D_{0} \quad$ diffusion coefficient of ions in water $\left(\mathrm{m}^{2} / \mathrm{s}\right)$.

$D^{\text {eff }} \quad$ effective diffusion coefficient of ions in porous media $\left(\mathrm{m}^{2} / \mathrm{s}\right)$.

$D^{\text {eff- } i} \quad$ predicted diffusion coefficient according a model $i\left(\mathrm{~m}^{2} / \mathrm{s}\right)$.

$D_{\max }^{\text {eff }} \quad$ upper bound of the effective diffusion coefficient $\left(\mathrm{m}^{2} / \mathrm{s}\right)$.

$D_{\mathrm{min}}^{\text {eff }} \quad$ lower bound of the effective diffusion coefficient $\left(\mathrm{m}^{2} / \mathrm{s}\right)$.

$D_{\mathrm{P}}^{1} \quad$ estimation of the diffusion coefficient in cement paste $\left(\mathrm{m}^{2} / \mathrm{s}\right)$.

$i \quad$ integer $(-)$.

I second-order unit tensor $(-)$.

*Present address: Laboratoire des Matériaux et des Structures du Génie Civil, LMSGC/LCPC, Marne la Vallée, France 
Greek Symbols

$\beta \quad$ tortuosity (-).

$\Phi \quad$ porosity (-).

\section{Introduction}

Chlorides, which are dissolved in the environment around a concrete structure can penetrate into it. Reinforcing steel in this structure begins to corrode when the chloride volume fraction at its level is high enough. The time to reach this critical volume fraction depends on the diffusion coefficient of chloride. This parameter is almost always determined experimentally for each concrete. The object of this study is to predict the value of this parameter, by considering the concrete properties due to its proportioning. The parameters, which have an effect on the chloride diffusion coefficient are the water to cement ratio (W/C) and the aggregates (Atkinson and Nickerson, 1984). The second section of this paper is a review of the determination of chloride diffusion coefficient at different scales. This review show that it is necessary to consider the concrete or the mortar as a three phase composite to match experimental observations. The third section introduces a new analytical model, using ' $n$ theoretical layers' around each inclusion (aggregate) for the diffusion coefficient. The fourth section of this paper shows the validation of this model, after experiments on various mortar specimens.

\section{Review of Models on Effective Chloride Diffusion Coefficient}

\subsection{DEFINITIONS}

For models of diffusion coefficient, which consider the structural properties of concrete, some definitions are to be given about the scales, which are taken into account. Cementitious materials are considered as saturated, so that the only process of chloride is diffusion. As a rule, three scales are considered (Figure 1):

- The microscopic scale $\left(10^{-9} \mathrm{~m}\right)$ takes into account the pore features. For cement-based materials, the transfer process is mainly the diffusion of ions through water-filled pores (Garboczy, 1990). These pores can be characterized by different parameters: porosity $\Phi$, tortuosity $\beta$.

- The mesoscopic scale $\left(10^{-6}-10^{-3} \mathrm{~m}\right)$ corresponds to a 'theoretically homogeneous' material including cement paste and aggregates. At this scale, the chloride diffusion coefficient depends on the characteristics of the cement paste and 


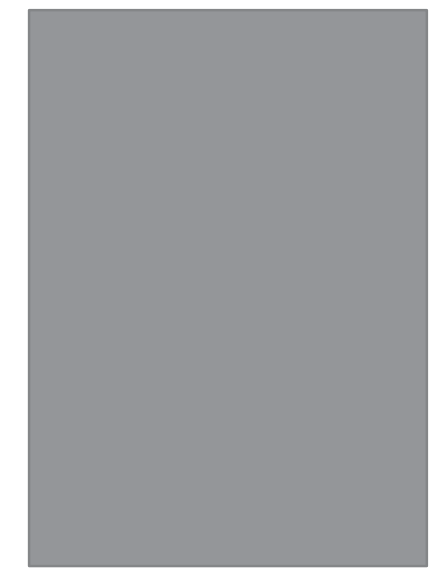

Macroscopic scale

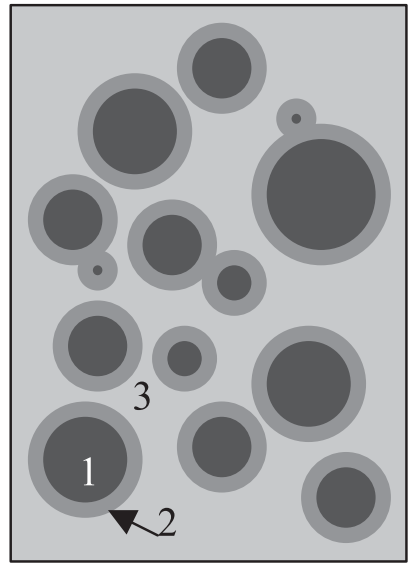

Mesoscopic scale

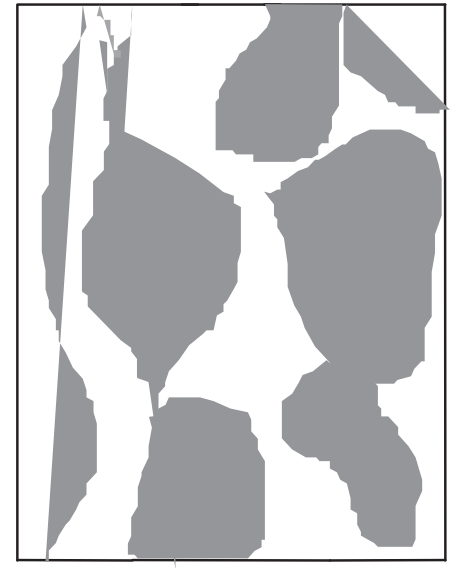

Microscopic scale

Figure 1. Scales used for studying diffusion problems. At macroscopic scale, the effective diffusion coefficient $D^{\text {eff }}$ is determined. At mesoscopic scale, $D^{\text {eff }}=f[C(i), D(i)]$, where $C(i)=$ volume fraction and $D(i)=$ diffusion coefficient of the different phases ( $1=$ aggregate, $2=$ interfacial transition zone and $3=$ bulk cement paste). At microscopic scale, $D^{\text {eff }}=f\left[D_{0}, \Phi, \beta\right]$, where $D_{0}=$ diffusion coefficient of ions in water and $\Phi=$ porosity and $\beta=$ tortuosity of pore space.

of the aggregates. More precisely, concrete may be considered as a composite material with three phases (Figure 1). The first one, related to the aggregates, is a dispersed phase with homogeneous properties. The aggregates can be porous (calcareous aggregates) or not (siliceous aggregates). The second phase, ITZ (interfacial transition zone), is an interface, with a gradient of porosity, and volume fractions gradients of anhydrous and hydrated cement compounds. The ITZ microstructure is different from that of the bulk cement paste due to the 'packing' constraints imposed by the aggregate surface (Garboczi and Bentz, 1997). The last phase is the bulk cement paste, a continuous phase also named matrix.

- The macroscopic scale (order of magnitude: $10^{-2} \mathrm{~m}$ ) corresponds to the size of specimens used for determining the effective chloride diffusion coefficient $D^{\text {eff }}\left(\mathrm{m}^{2} / \mathrm{s}\right)$ (Francy and François, 1998; François et al., 2001). This coefficient is obtained from the steady-state regime according to the first Fick's law:

$$
\vec{j}=D^{\mathrm{eff}} \overrightarrow{\mathrm{gradc}},
$$

where $\vec{j}$ represents the flux vector of free chloride ions $\left(\mathrm{kg} / \mathrm{m}^{2} \mathrm{~s}\right), c$ is the volume fraction $\left(\mathrm{kg} / \mathrm{m}^{3}\right)$ of free chloride ions. When inside the pore systems of concrete, chloride ions are partitioned between free ions that diffuse toward region with lower chloride concentration and bound chloride. $c$ is related to chloride-free ions, which do not interact with hydrated cement paste. The exchange between these different kind of ions is assumed to be instantaneous.

The steady-state regime is characterized by a linear concentration profile in the liquid phase between the upstream and the downstream cells. In this case, 
it is worth noticing that there is a local equilibrium between bound and free ions. It may be considered that there is no interaction between free chloride and solids phase. The second Fick's law reads then:

$$
\operatorname{div} \vec{j}=0 \text {, }
$$

\subsection{USUAL MODELS}

The usual models for chloride diffusion coefficient are as follows:

\subsubsection{Empirical Modeling at Macroscopic Scale}

Previous data show that the diffusion coefficient in cement paste is well correlated with the water to cement ratio. According to Atkinson and Nickerson (1984), in cement paste, the relationship between the chloride diffusion coefficient and the water to cement ratio, is approximately in an exponential one. According to Hobbs (1999), the diffusion coefficient in concrete depends on the water to cement ratio, but the study of this dependence shows that the correlation is weaker for concrete than for cement pastes. These results show that the effect of aggregates must be taken into account.

\subsubsection{Microscopic Scale Models}

As a rule, the effective diffusion coefficient in porous media $D^{\text {eff }}$ is lower than that of the same ions in water $D_{0}$. According to Garboczi (1990), Van Barkel and Heetjes (1974) and Dormieux and Lemarchand (2000) $D^{\text {eff }}$ is given by

$$
D^{\mathrm{eff}}=D_{0} \Phi \beta=D_{0} \frac{2 \Phi}{3-\Phi},
$$

where $\beta=2 /(3-\Phi)$ is the 'tortuosity' of the pore system. This result is obtained by an homogenization technique (Mori Tanaka's approach (Dormieux and Lemarchand, 2000) using Maxwell's model) and is an estimation of the effective diffusion coefficient of a composite material where the solid grains are considered as spherical inclusions in a fluid phase.

Furthermore, another multiscale modeling technique may be used for the determination of the effective diffusion coefficient. Xu et al. (1997) determine the diffusion coefficient by using the multiscale percolation system concept. In this case, the 'modeled material' is made of several elementary networks having mesh size proportional to the diameter of the real pores. The distribution of pore size is determined by mercury intrusion porosimetry.

These two kinds of models (Xu et al., 1997; Dormieux and Lemarchand, 2000) are applicable on any cement-based materials (cement paste, mortar, concrete). Nevertheless there is a poor agreement between the value predicted by these models and the experimental diffusion coefficients (Daian, 2001). As a rule, the predicted values are higher than the experimental ones. 


\subsubsection{Mesoscopic Scale Models}

At this scale, the model materials is a two-phase or three-phase composite. The models take into account the volume fractions of the components (aggregates, bulk cement matrix and interfacial transition zone ITZ) and their diffusive properties.

2.2.3.1. Two-Phase Models. In this case, the material (concrete, mortar) is a twophase composite, one phase is the homogeneous cement paste and the other phase includes aggregates. The volume fraction of the aggregates is $C_{\mathrm{A}}$ and that of the cement paste is $\left(1-C_{\mathrm{A}}\right)$. The diffusion coefficients are $D_{\mathrm{A}}$ for aggregate phase and $D_{\mathrm{P}}$ for cement paste phase. Various models are given in literature.

Hobbs (1997) proposed two models, which are based on the following assumptions: the decrease in chloride volume fraction and the flux (mass of chloride ions carried across a unit area) are the same in cement paste as in aggregates. The first assumption gives an upper bound for the effective diffusion coefficient ( $\left.D_{\max }^{\text {eff }}\right)$ and the second one a lower bound $\left(D_{\mathrm{min}}^{\mathrm{eff}}\right)$. These two models are similar to the Voigt and Reuss models used to bound the elastic modulus of two-phase materials (Hashin and Shrikman, 1963). The effective diffusion coefficient $D^{\text {eff }}$ is a function of $D_{\mathrm{P}}$, $D_{\mathrm{A}}$ and $C_{\mathrm{A}}$. The lower and upper bounds are estimated by

$$
D_{\text {min }}^{\text {eff }}=\frac{1}{C_{\mathrm{A}} / D_{\mathrm{A}}+\left(1-C_{\mathrm{A}}\right) / D_{\mathrm{P}}}, \quad D_{\max }^{\text {eff }}=\left(1-C_{\mathrm{A}}\right) D_{\mathrm{P}}+C_{\mathrm{A}} D_{\mathrm{A}} .
$$

When aggregates are not porous $\left(D_{\mathrm{A}}=0\right)$, the effective diffusion coefficient $D_{\min }^{\text {eff }}=0$ and $D_{\max }^{\text {eff }}$ is equal to

$$
D_{\max }^{\mathrm{eff}}=\left(1-C_{\mathrm{A}}\right) D_{\mathrm{P}} \text {. }
$$

Nevertheless, both relations given in Equation (4) correspond to a particular morphology: the two phases (aggregates and cement paste), have configurations in series or in parallel.

In the case of porous aggregates $\left(D_{\mathrm{A}} \neq 0\right)$, an estimation of the effective diffusion coefficient $D^{\text {eff }}$, which takes into account the matrix-aggregate configuration in the studied materials (aggregates considered as inclusions in the cement paste matrix) is given by $\mathrm{Xi}$ and Bazant (1999) according to Christensen (1979), and is equal to

$$
D^{\text {eff }}=D_{\mathrm{P}} \frac{2 D_{\mathrm{P}}\left(1-C_{\mathrm{A}}\right)+D_{\mathrm{A}}\left(1+2 C_{\mathrm{A}}\right)}{D_{\mathrm{P}}\left(2+C_{\mathrm{A}}\right)+D_{\mathrm{A}}\left(1-C_{\mathrm{A}}\right)}
$$

For non-porous aggregates $\left(D_{\mathrm{A}}=0\right)$, Equation (6) with $D_{\mathrm{A}}=0$ gives an estimation of $D^{\text {eff: }}$

$$
D^{\mathrm{eff}}=D_{\mathrm{P}} \frac{2\left(1-C_{\mathrm{A}}\right)}{2+C_{\mathrm{A}}} .
$$

It is worth noticing that model in Equation (7) is always Maxwell's model and may be obtained from Equation (3) by replacing the porosity $\Phi$ by the volume fraction of cement paste $\left(1-C_{\mathrm{A}}\right)$. 
2.2.3.2. Three-Phase Models. These models take into account the presence of an interface between bulk cement paste and aggregate (ITZ). Garboczi and Bentz (1998) proposes analytical and numerical models. By applying homogenization technique to a dilute material (named dilute limit), Garboczi (1990) determines the diffusion coefficient $D^{\text {eff }}$ as an analytical function of the diffusion coefficient in the bulk cement matrix $\left(D_{\mathrm{B}}\right)$, of the aggregates size distribution and of the geometrical and physical properties of ITZ. But, this approach is applicable only for very low aggregate volume fractions $\left(C_{\mathrm{A}} \ll 1\right)$. By using a differential effective medium theory, the result of the dilute limit is used to obtain the effective diffusion coefficient whatever the aggregate volume fraction. For this approach, a numerical resolution is necessary. Furthermore the same author (Garboczi and Bentz, 1998), with another numerical approach, puts the properties computed at one scale, micrometers for instance, into a model which is built at a higher scale, such as millimeters. The model proposed in Garboczi and Bentz (1998) combines microstructure models for the cement paste surrounding a single aggregate and for a representative volume of concrete.

Furthermore, Jaiswal et al. (1998) determine the diffusion coefficient by using non-linear statistical analysis and physically based approximate models. An analytical formulation of the composite diffusion coefficient is based on Bruggeman's (1935) effective medium theory with percolation effects by McLachlan et al. (1990). Jaiswal et al. (1998) show that the ITZ must be included in models to match experimental observations, although the ITZ cannot be directly measured and its existence is often questioned.

\subsection{SYNTHESIS OF THE REVIEW}

Several models have been published to predict the diffusion coefficient. Three scales are considered for these models. At the macroscopic scale, empirical models propose the diffusion coefficient to be a function of the water to cement ratio in the material. At the microscopic scale, analytical models propose the diffusion coefficient to be a function of the microstructure parameters. Nevertheless there is a poor agreement between the predicted value of the diffusion coefficient and the experimental one (Daian, 2001). At the mesoscopic scale, numerical and analytical models are proposed to determine the diffusion coefficient of mortar as a two-phase or a three-phase composite. Few analytical models taking into account the presence of ITZ are proposed, although various works show that the ITZ must be included in models (Jaiswal et al., 1998).

The aim of this work is to propose a new analytical model for chloride diffusion coefficient in mortar, which takes into account the characteristics of the material considered as a three-phase composite. In order to predict the effective chloride diffusion coefficients in concrete, the $(n+1)$-phase model, already used in elasticity, viscoelasticity, elastoplasticity (Hervé and Zaoui, 1993, 1995) and in the case 
of thermal and thermoelastic behaviors (Hervé, 2002), is extended to the case of ionic diffusion.

\section{Model for Diffusion Coefficient in a $\boldsymbol{n}$-Phase Material - Application to Mortar}

This section is split into two parts. In the first one, we show how we can, in order to predict the diffusion coefficient in a $n$-phase material, directly use the $(n+1)$-phase model (Hervé, 2002) originally proposed in the case of thermal and thermoelastic behaviors. The $(n+1)$-phase model is used to find an estimation of the effective diffusion coefficient of an $n$-layered-reinforced material. In the second part the $(n+1)$-phase model is finally used to determine the overall diffusion of chlorides in concrete where the first phase is the aggregate, the second one the interfacial transition zone with homogeneous properties and the third one the bulk cement paste. Two cases are considered: diffusion can or cannot take place in aggregates.

\subsection{THE $(n+1)$-PHASE MODEL}

To derive the ionic concentration field in an infinite medium constituted of an $n$-layered spherical inclusion, embedded in a matrix subjected to a uniform concentration rise at infinity (as partially done in Garboczi and Bentz (1996)), the ion volume fraction $c\left(\mathrm{~kg} / \mathrm{m}^{3}\right)$ compared to a base concentration is defined and as in Hervé and Zaoui (1993) the following infinite medium, which has $n$ isotropic phases, is considered. Phase 1 constitutes the central core and phase (i) lies within the shell limited by the two concentric spheres with the radii $R_{i-1}$ and $R_{i}$. Let $\left(\mathbf{D}=D_{i} \mathbf{I}\right)$ be the diffusion tensor of phase (i) (here $i[1, n+1], R_{0}=0$ and $R_{n+1} \rightarrow \infty$ ) (Figure 2), in which $\mathbf{I}$ is the second-order unit tensor (each phase is
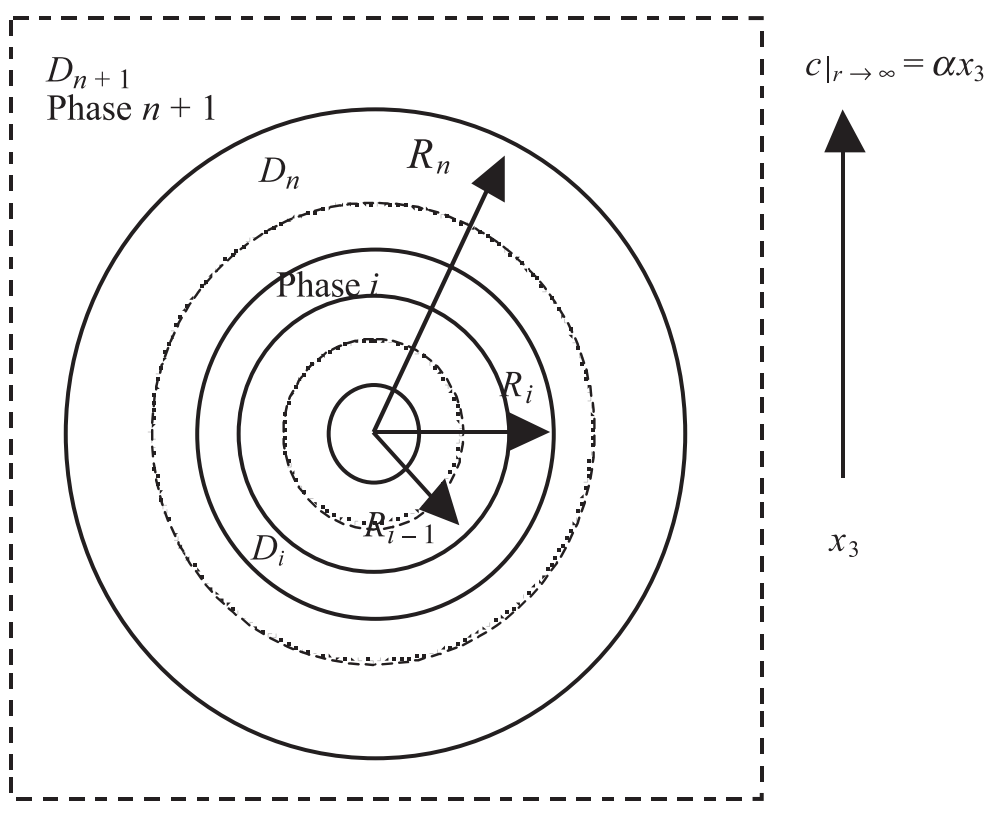

Figure 2. A $n$-layered spherical inclusion in an infinite medium. 
isotropic). The spherical coordinate system $(r, \theta, \varphi)$ has its origin at the common center of the above-mentioned spheres The axial symmetry is around $x_{3}$-axis $\left(x_{3}=r \cos \theta\right)$. At infinity, the imposed volume fraction is given by

$$
c_{\mid r \rightarrow \infty}=\alpha x_{3},
$$

where $x_{k}$ are Cartesian coordinates and $\alpha$ is a scalar.

Each phase obeys the first Fick's law of ionic diffusion:

$$
\vec{j}=-D \overrightarrow{\operatorname{grad} \mathrm{c}}
$$

where $\vec{j}$ is the flux vector in each phase.

There is no source or sink of ions and under steady state conditions we can use Equation (2).

Both Equations (9) and (2) lead to the governing equation $\left(\Delta c^{(k)}=0\right)$ for $c^{(k)}$ in each phase $k$. The general solution of this governing equation which respects the imposed remote condition (8) is given in phase $(k)$ by

$$
c^{(k)}=\left(A_{k} r+\frac{B_{k}}{r^{2}}\right) \cos \theta,
$$

where $A_{k}$ and $B_{k}$ are constants, $B_{1}=0$ to avoid singularity at the origin and $A_{n+1}=\alpha$ accounting for the imposed remote condition (it is worth noticing that in the case of a remote flux-type condition $\vec{j}=-\gamma \vec{e}_{3}, A_{n+1}=\gamma / D_{n+1}$ ).

The components of the corresponding flux vector are:

$$
\begin{aligned}
& j_{r}^{(i)}=-D_{i}\left(A_{i}-2 \frac{B_{i}}{r^{3}}\right) \cos \theta, \quad j_{\theta}^{(i)}=+D_{i}\left(A_{i}+\frac{B_{i}}{r^{3}}\right) \sin \theta, \\
& j_{\varphi}^{(i)}=0 .
\end{aligned}
$$

From the two conditions describing the continuity of $j_{r}$ and $c$ at the interface $r=$ $R_{k}$ between phases $(k)$ and $(k+1)$ (Hervé, 2002) all the coefficients $A_{k}$ and $B_{k}(k \in$ $[1, n])$ can be expressed in terms of the diffusion coefficient of each phase, their volume fraction and $A_{n+1}$.

From these results the third component of the average ion volume fraction gradient is calculated in the whole $n$-layered inclusion:

$$
\langle c, 3\rangle=\frac{1}{V_{n}} \int_{V_{n}} c_{, 3} \mathrm{~d} V=\left(A_{n+1}+\frac{B_{n+1}}{R_{n}^{3}}\right) .
$$

$V_{n}$ is the volume of this $n$-layered inclusion. Correspondingly, the third component of the average flux vector of ions in the whole $n$-layered inclusion reads:

$$
\left\langle j_{3}\right\rangle=-D_{n+1}\left(A_{n+1}+\frac{B_{n+1}}{R_{n}^{3}}\right) .
$$

These solutions can be used if a true $n$-phase inclusion is considered in a welldefined matrix whose diffusion coefficient $D_{n+1}$ is known. It can also be used if $D_{n+1}$ (named then $D^{\text {eff }}$ ) denotes the effective diffusion coefficient of a composite material according to the $(n+1)$-phase model. 
To determine the effective diffusion coefficient of an isotropic composite material according to the ' $(n+1)$-phase model', one of the two self-consistent conditions can be used. They both lead to relation $B_{n+1}=0$. The first possible condition is to impose the third component of the average concentration gradient in the whole $n$-layered inclusion to be the same as the third component of the macroscopic concentration gradient imposed to the composite medium at infinite $\left(\left\langle c_{, 3}\right\rangle=\alpha\right)$. The second possible condition is to write that the third component of the average ion flux vector in the whole $n$-layered inclusion is the same as the third component of the ion flux vector imposed to the composite medium at infinity $\left\langle j_{3}\right\rangle=-\gamma$.

Setting $B_{n+1}=0$ provides the effective diffusion coefficient (Hervé, 2002) given hereafter by a recursive algorithm:

$$
D_{(i)}^{\mathrm{eff}}=D_{i}+\frac{D_{i}\left(R_{i-1}^{3} / R_{i}^{3}\right)}{\left(D_{i} /\left(D_{(i-1)}^{\mathrm{eff}}-D_{i}\right)\right)+(1 / 3)\left(\left(R_{i}^{3}-R_{i-1}^{3}\right) / R_{i}^{3}\right)},
$$

where $D_{(1)}^{\text {eff }}=D_{1}$.

\subsection{APPLICATION TO THE DIFFUSION COEFFICIENT OF CHLORIDE IN CONCRETE}

These results are applied to the problem of diffusion of chlorides in concrete. As in Garboczi and Bentz (1996), a four-phase model is used. Phase 1 denotes the aggregates (considered as spherical inclusions) with the volume fraction $C_{\mathrm{A}}$ and the diffusion coefficient $D_{\mathrm{A}}$. Phase 2 denotes the interfacial transition zone with the volume fraction $C_{\mathrm{I}}$ and the diffusion coefficient $D_{\mathrm{I}}$. Phase 3 denotes the bulk cement paste with the diffusion coefficient $D_{\mathrm{B}}$. To find the effective diffusion coefficient in concrete we use Equation (14) and we proceed in three steps:

(a) We consider an homogeneous material made of aggregates:

$$
D_{(1)}^{\text {eff }}=D_{\mathrm{A}} \text {. }
$$

(b) We consider a two-phase material made of inclusions (aggregates) embedded in a matrix (ITZ):

where

$$
D_{(2)}^{\mathrm{eff}}=D_{1}+\frac{D_{1}\left(R_{1}^{3} / R_{2}^{3}\right)}{\left(D_{\mathrm{I}} /\left(D_{\mathrm{A}}-D_{\mathrm{I}}\right)+(1 / 3) /\left(\left(R_{2}^{3}-R_{1}^{3}\right) / R_{2}^{3}\right)\right.},
$$

$$
\frac{R_{2}^{3}-R_{1}^{3}}{R_{2}^{3}}=\frac{C_{\mathrm{I}}}{C_{\mathrm{A}}+C_{\mathrm{I}}}, \quad \frac{R_{1}^{3}}{R_{2}^{3}}=\frac{C_{\mathrm{A}}}{C_{\mathrm{A}}+C_{\mathrm{I}}} .
$$

(c) We consider coated inclusions (aggregates surrounded by the ITZ) embedded in a matrix (bulk cement paste):

$$
D_{(3)}^{\text {eff }}=D_{\mathrm{B}}+\frac{D_{\mathrm{B}}\left(R_{2}^{3} / R_{3}^{3}\right)}{\left(D_{\mathrm{B}} /\left(D_{(2)}^{\mathrm{eff}}-D_{\mathrm{B}}\right)\right)+(1 / 3)\left(\left(R_{3}^{3}-R_{2}^{3}\right) / R_{3}^{3}\right)},
$$

where

$$
\frac{R_{3}^{3}-R_{2}^{3}}{R_{3}^{3}}=1-C_{\mathrm{A}}-C_{\mathrm{I}}, \quad \frac{R_{2}^{3}}{R_{3}^{3}}=C_{\mathrm{A}}+C_{\mathrm{I}} .
$$


Finally, substituting for $D_{2}^{\text {eff }}$ from Equation (16) into Equation (18) and taking Equation (15) into account yields

$$
\frac{D^{\mathrm{eff}}}{D_{\mathrm{B}}}=\frac{N}{D}
$$

where

$$
\begin{aligned}
N= & 6 D_{\mathrm{B}} D_{\mathrm{I}}\left(1-C_{\mathrm{A}}\right)\left(C_{\mathrm{A}}+C_{\mathrm{I}}\right)+C_{\mathrm{I}}\left(1+2 C_{\mathrm{A}}+2 C_{\mathrm{I}}\right)\left(D_{\mathrm{I}}-D_{\mathrm{B}}\right) \times \\
& \times\left(2 D_{\mathrm{I}}+D_{\mathrm{A}}\right)+3 D_{\mathrm{A}}\left[C_{\mathrm{I}} D_{\mathrm{B}}+C_{\mathrm{A}} D_{\mathrm{I}}\left(1+2 C_{\mathrm{A}}+2 C_{\mathrm{I}}\right)\right],
\end{aligned}
$$

and

$$
\begin{aligned}
D= & 3 D_{\mathrm{B}} D_{\mathrm{I}}\left(2+C_{\mathrm{A}}\right)\left(C_{\mathrm{A}}+C_{\mathrm{I}}\right)+C_{\mathrm{I}}\left(1-C_{\mathrm{A}}-C_{\mathrm{I}}\right)\left(D_{\mathrm{I}}-D_{\mathrm{B}}\right) \times \\
& \times\left(2 D_{\mathrm{I}}+D_{\mathrm{A}}\right)+3 D_{\mathrm{A}}\left[C_{\mathrm{I}} D_{\mathrm{B}}+C_{\mathrm{A}} D_{\mathrm{I}}\left(1-C_{\mathrm{A}}-C_{\mathrm{I}}\right)\right] .
\end{aligned}
$$

The relationship (20) allows us to investigate materials with porous aggregates and with ITZ. Furthermore from this relation, several cases of concrete may be considered too. Indeed, this investigation makes it possible to identify the relevant parameters, which affect chloride transport in concrete with porous or non-porous aggregates and with or without ITZ.

When there is no ITZ $\left(C_{\mathrm{I}}=0\right.$ or $\left.D_{\mathrm{I}}=D_{\mathrm{B}}=D_{\mathrm{P}}\right)$ and when aggregates are porous $\left(D_{\mathrm{A}} \neq 0\right)$, relationship (20) is equivalent to Equation (6). When the diffusion coefficient of the aggregates is $D_{\mathrm{A}}=0$ and when ITZ is absent $\left(C_{\mathrm{I}}=\right.$ 0 or $D_{\mathrm{I}}=D_{\mathrm{B}}=D_{\mathrm{P}}$ ), relationship (20) is equivalent to Equation (7). In this case, $D_{\mathrm{P}}$ is the diffusion of cement paste $\left(D_{\mathrm{B}}\right)$. Furthermore, when the diffusion coefficient of the aggregates vanishes $\left(D_{\mathrm{A}}=0\right)$, the relationship (20) reads:

$$
D^{\mathrm{eff}}=D_{\mathrm{B}} \frac{6 D_{\mathrm{B}}\left(1-C_{\mathrm{A}}\right)\left(C_{\mathrm{A}}+C_{\mathrm{I}}\right)+2 C_{\mathrm{I}}\left(D_{\mathrm{I}}-D_{\mathrm{B}}\right)\left(1+2 C_{\mathrm{A}}+2 C_{\mathrm{I}}\right)}{3 D_{\mathrm{B}}\left(2+C_{\mathrm{A}}\right)\left(C_{\mathrm{A}}+C_{\mathrm{I}}\right)+2 C_{\mathrm{I}}\left(1-C_{\mathrm{A}}-C_{\mathrm{I}}\right)\left(D_{\mathrm{I}}-D_{\mathrm{B}}\right)} .
$$

\section{Validation of the Model}

\subsection{MATERIALS AND EXPERIMENTAL PROCEDURES}

The model of the diffusion coefficient of chloride in concrete given in Equation (21) is validated by experiments on specimens. The materials tested are one cement paste $(\mathrm{P})$ and five mortars (M1C, M2C, M2M, M1F, M2F) with non-porous aggregates $\left(D_{\mathrm{A}}=0\right)$. Table I gives their compositions. Three different types of aggregates are used: a fine siliceous sand $(0.315 / 1 \mathrm{~mm})$ labeled ' $\mathrm{F}$ ', a medium siliceous sand $(0.315 / 2 \mathrm{~mm})$ labeled ' $M$ ' and a coarse siliceous sand $(2 / 4 \mathrm{~mm})$ labeled ' $C$ '. These compositions make it easy to study the influence of the interfacial transition zones on chloride diffusion process. The water to cement ratio $(\mathrm{W} / \mathrm{C}=0.45)$ and the type of cement (coarse cement) are chosen so that the microstructure of the 
Table I. Composition of the tested cement paste and mortars

\begin{tabular}{lllllll}
\hline & \multicolumn{2}{l}{ Mixture } & & & & \\
\cline { 2 - 7 } & $\mathrm{P}$ & $\mathrm{M} 1 \mathrm{C}$ & $\mathrm{M} 2 \mathrm{C}$ & $\mathrm{M} 2 \mathrm{M}$ & $\mathrm{M} 1 \mathrm{~F}$ & $\mathrm{M} 2 \mathrm{~F}$ \\
\hline W/C & 0.45 & 0.45 & 0.45 & 0.45 & 0.45 & 0.45 \\
Aggregate & $/$ & $\mathrm{C}$ & $\mathrm{C}$ & $\mathrm{M}$ & $\mathrm{F}$ & $\mathrm{F}$ \\
$C_{\mathrm{A}}$ & 0 & 0.25 & 0.5 & 0.5 & 0.25 & 0.5 \\
\hline
\end{tabular}

interfacial transition zone differs significantly from that of the bulk matrix. These mortars compositions studied are far from standard composition but they allow to validate model.

The experimental procedures and the results obtained are developed in Caré (2003). Chloride-effective diffusion coefficient $D^{\text {eff }}$ is determined from non-steadystate diffusion tests by applying Fick's second law after determining for each material its total chloride profile and its chloride binding isotherm (Francy and François, 1996; François et al., 2001). The microstructure of these materials is observed by scanning electron microscopy (SEM) and mercury intrusion porosimetry (MIP) is used to characterize the pore structure.

\subsection{RESULTS}

\subsubsection{Interfacial Transition Zone Characteristics}

The results obtained by MIP for the total porosity are summarized in Table II. For mortars, the finer is the sand used, the higher is the total porosity. The presence of aggregates changes the pore structure too (Caré, 2003). First, in the presence of aggregates, cement paste contains more fine pores. Second, pores appear with a size larger than what is observed in cement paste without aggregates and may be attributed to ITZ. These data agree with previously published results (Bentur and Alexander, 2000): a more porous ITZ is observed when bulk cement paste is more dense (due to a lower value of the water to cement ratio). Furthermore, SEM observations (Caré, 2003) show that aggregates are surrounded by an interface between

Table II. Data on the microstructure of the tested materials

\begin{tabular}{lllcccc}
\hline & \multicolumn{2}{l}{ Mixture } & & & & \\
\cline { 2 - 6 } & $\mathrm{P}$ & $\mathrm{M} 1 \mathrm{C}$ & $\mathrm{M} 2 \mathrm{C}$ & $\mathrm{M} 2 \mathrm{M}$ & $\mathrm{M} 1 \mathrm{~F}$ & $\mathrm{M} 2 \mathrm{~F}$ \\
\hline$\Phi(\%)$ & 22.6 & 15.1 & 11 & 12.2 & 17.9 & 13 \\
$C_{\mathrm{I}}(\%)$ & $/$ & 1.8 & 3.6 & 10.3 & 8 & 15.4 \\
\hline
\end{tabular}


aggregate and bulk cement paste (ITZ). The thickness $\left(t_{\mathrm{I}}\right)$ of ITZ is measured by SEM and is about $30 \mu \mathrm{m}$, depends on the type of cement and not on the aggregate size distribution. The ITZ volume fraction $C_{\mathrm{I}}$ is evaluated according to Garboczi and Bentz (1997):

$$
C_{\mathrm{I}}=\left(1-C_{\mathrm{A}}\right)-\left(1-C_{\mathrm{A}}\right) e_{\mathrm{V}}\left(t_{\mathrm{I}}\right) .
$$

The function $e_{\mathrm{V}}\left(t_{\mathrm{I}}\right)$ depends on aggregate volume fraction, on aggregate size distribution and on ITZ thickness (Garboczi and Bentz, 1997). Equation (22) takes into account possible overlaps of interfacial transition zones. Table II shows the ITZ volume fraction $C_{\mathrm{I}}$ for the studied mortars.

\subsubsection{Effective Chloride Diffusion Coefficient $D^{\text {eff }}$}

Table III presents the experimental results concerning $D^{\text {eff }}$, the diffusion coefficients for the studied materials. The errors on the experimental values of diffusion coefficients $D^{\text {eff }}$ are estimated to be $30 \%$ (Caré, 2003). It appears that the diffusion coefficient $D^{\text {eff }}$ depends on the aggregate volume fraction and on the aggregate size distribution.

The influence of aggregate properties can be evaluated by the estimation of the diffusion coefficient in the cement paste (named $D_{\mathrm{P}}^{1}$ ) of the mortars. To determine $D_{\mathrm{P}}^{1}$, some authors (Delagrave et al., 1997) used the upper value $D_{\max }^{\text {eff }}$ as an estimation of the effective diffusion coefficient in Equation (5). But this equation does not take into account the matrix-aggregate configuration in the studied materials, as shown previously, so $D_{\mathrm{P}}^{1}$ is estimated by reversing Equation (7). $D_{\mathrm{P}}^{1}$ is given in Table III. The diffusion coefficient $D_{\mathrm{P}}^{1}$ of the cement paste of the mortars is higher than that of the cement paste $\mathrm{P}$. The influence of aggregates on the diffusion coefficient $D_{\mathrm{P}}^{1}$ may be attributed to the presence of the porous ITZ in the materials.

So, it is interesting to compare the diffusion coefficient for interfacial transition zone $D_{\mathrm{I}}$ with that for bulk cement paste $D_{\mathrm{B}}$. The diffusion coefficient for ITZ $D_{\mathrm{I}}$ is evaluated for the five mortars, by reversing Equation (21), where $C_{\mathrm{A}}, C_{\mathrm{I}}$ are determined from the mixture proportions. The diffusion coefficient $D_{\mathrm{B}}$ is assumed to be always equal to that of plain cement paste $\mathrm{P}$ (without aggregate), although the assumption concerning a constant value for $D_{\mathrm{B}}$ is not always exactly valid because the presence of aggregates changes the porosity of cement paste even far from the interfacial zone. The obtained values of ITZ diffusion coefficient $D_{\mathrm{I}}$ are given in Table III. The ratio $D_{\mathrm{I}} / D_{\mathrm{B}}$ appears to decrease with increasing ITZ volume fraction $C_{\mathrm{I}}$. This result may be explained by the assumption on the diffusion coefficient $D_{\mathrm{B}}$ which may be overestimated for mortar with high ITZ volume fraction (their bulk cement paste is more dense than the plain cement paste P).

This analysis confirms that the cement paste in mortar includes two phases with different diffusive properties. 


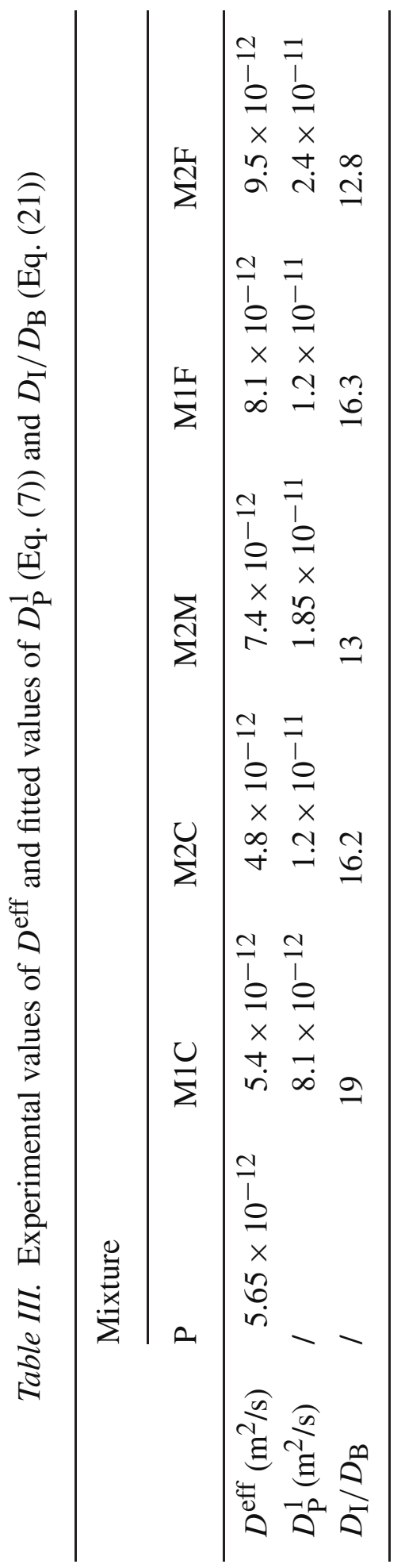




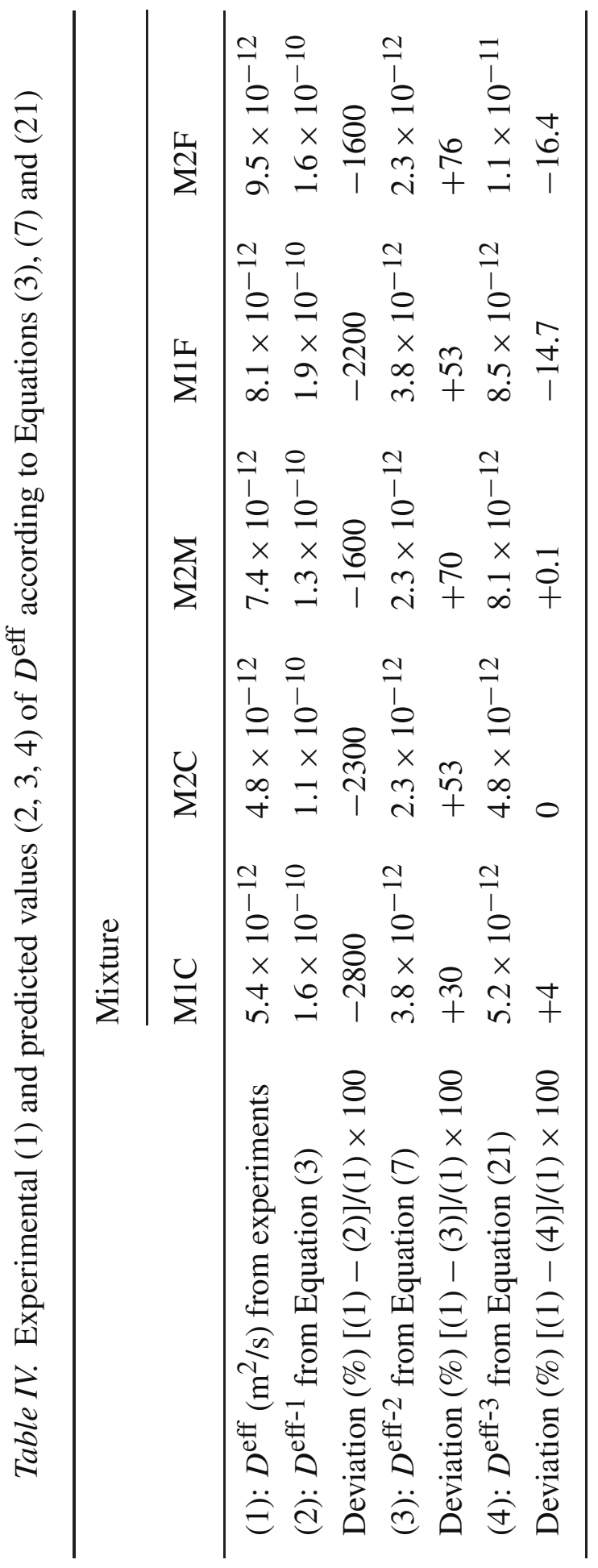




\subsection{MODELING OF THE EFFECTIVE DIFFUSION COEFFICIENT}

The modeling of the effective diffusion coefficient is considered at different scales. Predicted values of the effective diffusion coefficient are given in Table IV according to different models at microscopic and mesoscopic scales.

Experimental results show that the effective diffusion coefficient depends on the aggregate properties (volume fraction and size) and cannot be modeled, at macroscopic scale, only by the water to cement ratio as shown by Hobbs (1990).

If microscopic scale model (Eq. (3) with $D_{0}=1.5 \times 10^{-9} \mathrm{~m}^{2} / \mathrm{s}$ and $\Phi$ given in Table II) is considered, the predicted values (named $D^{\mathrm{eff}-1}$ ) are higher than the experimental ones. This result shows that the microscopic model is not valid according to Daian (2001).

If mesoscopic scale model is considered, the mortars may be considered as a two-phase composite or a three-phase composite. In a first step, the mortars are considered as a two-phase composite without ITZ. In this case, the diffusion coefficient in the cement paste is assumed to be equal to that of cement paste $\mathrm{P}$ (without aggregate). The predicted values of $D^{\text {eff }}$ (named $D^{\text {eff-2 }}$ ) are given in Table IV. The difference between the predicted values $D^{\text {eff-2 }}$ and the experimental coefficients is higher than the experimental uncertainties. So, this result confirms that the mortars have to be considered as a three-phase composite. The effective diffusion coefficient have to be estimated from the value of the diffusion coefficient in the interfacial transition zone $D_{\mathrm{I}}$ and that of the bulk cement paste $D_{\mathrm{B}}$. The diffusion coefficient of the bulk cement paste is assumed to be that of the cement paste $\mathrm{P}$ without aggregate. As the diffusion coefficient of ITZ $D_{\mathrm{I}}$ cannot be directly measured (Jaiswal et al., 1998), $D_{\mathrm{I}}$ is evaluated from the result of the mortar M2C given in Table III. An only value for $D_{\mathrm{I}} / D_{\mathrm{B}}$ is considered, although this ratio given in Table III is not the same for all mortars. When $D_{\mathrm{I}}=16.2 D_{\mathrm{B}}$, the coefficients for the mortars M1C, M2M, M1F and M2F (named $D^{\text {eff-3) }}$ ) are estimated without fitting. Calculated coefficients are presented in Table IV. The difference between the test results and the predicted results is small, suggesting that the proposed model is suitable for the effective diffusion coefficient because they are less than the uncertainties on the values $(30 \%)$.

These comparisons suggest that the model given by Equation (21) is the best one to estimate the effective diffusion coefficient of mortar. So, a procedure to estimate the diffusion coefficient $D^{\text {eff }}$ of chloride in mortar can be as follows. When this diffusion coefficient is known for the related cement paste (without aggregate) and for a reference mortar, it can be estimated for any other mortars, with any aggregate volume fraction and any aggregate size distribution.

\section{Conclusions}

A new model based on ' $n$-layered inclusion' is established and used to predict the effective diffusion coefficient of chlorides in mortar or concrete. The material is composed of three phases: the first phase is aggregates, the second one the inter- 
facial transition zone and the third one the bulk cement paste. In this case, the model used is called a four-phase model. This model is validated by experiments on specimens made of plain cement paste and of mortar. The following conclusions can be drawn. The relevant factors that affect chloride transport in mortar are the aggregates volume fraction $C_{\mathrm{A}}$ and the volume fraction of interfacial transition zone, ITZ. The effective diffusion coefficients in the bulk cement paste and in ITZ depend on the mixture proportions of cement paste (water to cement ratio).

According to this model, the effective diffusion coefficient of chloride depends on the mixture parameters which are:

- The volume fractions $C_{\mathrm{A}}$, for aggregates and $C_{\mathrm{I}}$, for ITZ. The latter parameter $C_{\mathrm{I}}$ depends on the aggregate volume fraction, on the size distribution and on this ITZ thickness (the thickness is controlled by the cement type).

- The diffusion coefficients $D_{\mathrm{B}}$, for bulk cement paste and $D_{\mathrm{I}}$, for ITZ can be determined directly and after fitting the results.

So the main advantage of the proposed model is its simplicity for predicting the effective diffusion coefficient of mortar. It means that it is easily applicable. But, for practical applications, it has to be improved because the thickness of interfacial transition zone must be known for any cement-aggregate couple. A further validation with porous aggregates will be of interest.

\section{References}

Atkinson, A. and Nickerson, A. K.: 1984, The diffusion of ions through water-saturated cement, J. Mater. Sci. 19, 3068-3078.

Bentur, A. and Alexander, M. G.: 2000, A review of the work of the Rilem TC 159-ETC, Engineering of the interfacial transition zone in cementitious composites, Mater. Struct. 33, 82-87.

Bruggeman, D. A.: 1935, Ann. Phys. 24, 636.

Caré, S.: 2003, Influence of aggregates on chloride diffusion coefficient into mortar, Cem. Concr. Res. 33, 1021-1028.

Christensen, R. M.: 1979, Mechanics of Composite Materials, Wiley Interscience, New York.

Daian, J. F.: 2001, Evaluation des propriétés de transfert dans les matériaux cimentaires. Etude critique des modèles, Revue Française de Génie Civil, 5(2-3), 179-202.

Delagrave, A., Bigas, J. P., Ollivier, J. P., Marchand, J. and Pigeon, M.: 1997, Influence of the interfacial zone on the chloride diffusivity of mortars, Adv. Cem. Bas. Mat. 5, 86-92.

Dormieux, L. and Lemarchand, E.: 2000, Modélisation macroscopique du transport diffusif, apport des méthodes de changement d'espace, Oil Gas Sci. Technol. - Rev. IFP 55(1), 15-34.

François, R., Francy, O., Caré, S., Baroghel Bouny, V., Lovera, P. and Richet, C.: 2001, Mesure du coefficient de diffusion des ions chlorures: comparaison régime permanent-régime transitoire, Revue Française de Génie Civil 5(2-3), 309-329.

Francy, O. and François, R.: 1998, Measuring chloride diffusion coefficients from non-steady from non-steady state diffusion tests, Cem. Concr. Res. 28, 947-953.

Garboczi, E. J.: 1990, Permeability, diffusivity and microstructural parameters: a critical review, Cem. Concr. Res. 20, 591-601.

Garboczi, E. J. and Bentz, D. P.: 1996, The effect of the interfacial transition zone on concrete properties: the dilute limit, American Society of Civil Engineers, in: Proceedings of the Fourth Materials Conference, November 1996, Washington, DC. 
Garboczi, E. J. and Bentz, D. P.: 1997, Analytical formulas for interfacial transition zone properties, Adv. Cem. Bas. Mat. 6, 99-108.

Garboczi, E. J. and Bentz, D. P.: 1998, Multiscale analytical/numerical theory of the diffusivity of concrete, Advn. Cem. Bas. Mat. 8, 77-88.

Hashin, Z. and Shrikman, S.: 1963, A varationnal approach to the theory of the elastic behaviour of multi-phases materials, J. Mech. Phys. Solids. 11, 127-140.

Hervé, E.: 2002, Thermal and thermoelastic behaviour of multiply coated inclusion-reinforced composites, Int. J. Solids Struct. 39, 1041-1058.

Hervé, E. and Zaoui, A.: 1993, $N$-layered inclusion-based micromechanical modelling, Int. Eng. Sci. 31(1), 1-10.

Hervé, E. and Zaoui, A.: 1995, Elastic behaviour of multiply coated fibre-reinforced composites, Int. Eng. Sci. 33(10), 1419-1433.

Hobbs, D. W.: 1999, Aggregate influence on chloride ion diffusion into concrete, Cem. Concr. Res. 29, 1995-1998.

Jaiswal, S. S., Picka, J., Igusa, T., Ankenman, B., Karr, A. and Shah, S. P.: 1998, Impact of the interfacial transition zone on the chloride permeability of concrete, in: Proceedings of the 12th ASCE Congress, San Diego, May 17-20, 1998.

McLAchlan, D. S., Blaszkiewicz, M. and Newhham, R. E.: 1990, Electrical resistivity of composites, J. Am. Ceram. Soc. 73, 2187-2203.

Van Brakel, J. and Heetjes, P. M.: 1974, Analysis of diffusion in macroporous media in terms of a porosity, a tortuosity and a constrictivity factor, Int. J. Heat Mass Transfer 17, 1093-1103.

Xi, Y. and Bazant, Z. P.: 1999, Modeling chloride penetration in saturated concrete, J. Mater. Civil Eng., 58-65.

Xu, K., Daian, J. F. and Quénard, D.: 1997, Multiscale structures to describe porous media. Part II. Transport properties and application to test materials, Transport in Porous Media 26, 319-338. 